\title{
Ultrasonic Wave Propagation Analysis for In-Process Monitoring of Stamping
}

\author{
Naoto Hagino ${ }^{1, a^{*}}$, Seiji Komiya ${ }^{1, b}$, Junichi Endou ${ }^{1, c}$ and Masao Ishihama ${ }^{1, d}$ \\ ${ }^{1}$ Department of Vehicle System Engineering, Kanagawa Institute of Technology, \\ 1030, Shimo-ogino, Atsugi, Kanagawa 243-0292, Japan \\ anao_hagino@goo.jp, bkom@eng.kanagawa-it.ac.jp, ${ }^{\mathrm{c} e n d o @ s d . k a n a g a w a-i t . a c . j p, ~}$ \\ ishihama@sd.kanagawa-it.ac.jp
}

Keywords: In-process Monitoring, Stamping, Ultrasonic Wave, FDTD Method, Velocity Potential

\begin{abstract}
The servo press has high potential for producing high precision mechanical parts. However, small gaps between dies and workpieces tend to exist even in servo press stamping, and the potential of the servo press has not yet been fully utilized. The reason for this is conventional presses do not have feedback control systems, and the lack of a suitable method of sensing contact information in real time causes deterioration in the accuracy of products. If slide motion could be controlled by contact information, the small gaps could be removed. To solve this problem, the authors have developed a method of monitoring the contact states between dies and workpieces during the stamping process. The method uses ultrasonic wave reflection and transmission at the contact surfaces and was proved to be able to monitor contact pressure by using a simple geometry experimental die apparatus. Finite-difference time-domain (FDTD) numerical simulation was conducted in this study to obtain better understanding of wave propagation through dies and workpieces. The results obtained from this FDTD simulation visualized wave propagation that could not be experimentally measured. Some of the major results obtained are as follows. 1) When a thin metal sheet is pressed between dies that have inclined stamping surfaces, ultrasonic elastic waves are reflected and transmitted multiple times. 2) Modal conversion occurs at the die-workpiece boundary in such a way that normal waves with an inclined incident angle are transformed into normal and shear waves. 3) Elastic waves sent out from an ultrasonic transducer are mixtures of normal waves with flat wave fronts along the propagation path axis, normal waves with circular or spherical wave fronts expanding from both sides of the transducer, and shear waves. These results brought about much useful information for setting ultrasonic transducers and analyzing collected signals.
\end{abstract}

\section{Introduction}

Although servo presses have excellent potential for producing high precision mechanical parts, it is widely known that during stamping small gaps exist between the dies and workpieces. These gaps are mainly caused by inadequate positioning accuracy of the workpieces and the dies and by elastic deformation of the dies and the press slide. The main reason that the gaps are not removed during stamping is that the presses are not controlled by contact information. To achieve so-called "net-shape forming", the die shape must be precisely copied to the workpiece. If the slide motion could be controlled by contact information, the small gaps could be removed. It is thus necessary to develop methods for measuring the contact states between the workpiece and the die. The pressure between the tool and the workpiece can be measured by using ultrasonic waves [1], and the contact area between a mirror surface and a regular surface can be estimated by using reflected waves [2]. Additionally, an ultrasonic method has been used to measure the contact status between a bearing and a bearing housing [3]. A method using ultrasonic waves for measuring the contact states between the workpiece and the die during stamping was proposed in our previous studies [4-6]. The reflected and transmitted wave height could be used with this method to measure the contact states between the workpiece and the die. Additionally, we examined propagations of ultrasonic waves near the boundary surface with nominal analysis $[7,8]$. The sound fields in stamping dies were obtained by using the finite-difference time-domain (FDTD) method. Through FDTD simulation we were able to visualize wave propagation that could not experimentally be measured [7, 8]. However, the actual 
products have complicated shapes that consist of inclined or curved surfaces. When ultrasonic waves obliquely enter the workpiece, modal conversion of elastic waves occurs at the boundary surface of the workpiece and dies. Therefore, the acoustic fields near the boundary surface are complicated. Hence, the propagation behavior of ultrasonic waves on the boundary surfaces of workpieces and stamping dies was numerically analyzed by FDTD simulation in the present study. We used this to visualize the acoustic fields. The simulation results allowed us to visualize longitudinal and shear wave propagations that could not be experimentally measured.

\section{Principle of In-process Monitoring for Stamping}

Fig. 1 illustrates the propagation of ultrasonic waves at the boundary between two materials. The ultrasonic waves are transmitted into material 1 from an ultrasonic probe. The waves are either reflected or transmitted on the surface of the boundary between the two materials. The ratio of the waves reflected is called the "sound pressure reflectance" [1], which is given by:

$$
|R|=\left|\frac{Z_{1}-Z_{2}}{Z_{1}+Z_{2}}\right| .
$$

where $\mathrm{Z}$ is the acoustic impedance and subscripts 1 and 2 correspond to materials 1 and 2 ; here, $\mathrm{Z}$ is defined by:

$$
Z=\rho \times c_{l} .
$$

where $\rho$ is density and $c_{l}$ is the acoustic velocity of the longitudinal elastic waves in the material.

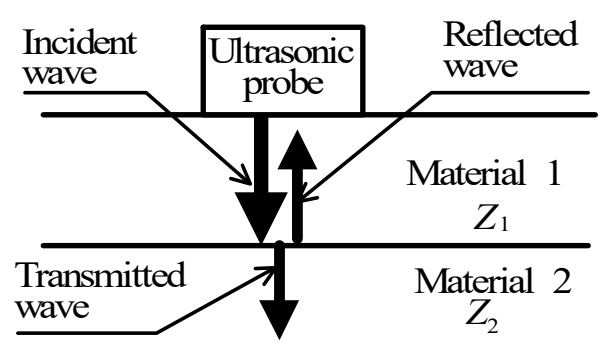

Fig. 1 Propagation of ultrasonic waves at boundary between different materials.

If $Z_{2}$ is far smaller than $Z_{1}, R$ is approximately one. For example, if materials 1 and 2 are steel and air, $R$ is almost 1 , which means that we should be able to measure the change in the gap between a workpiece and the die by using ultrasonic waves.
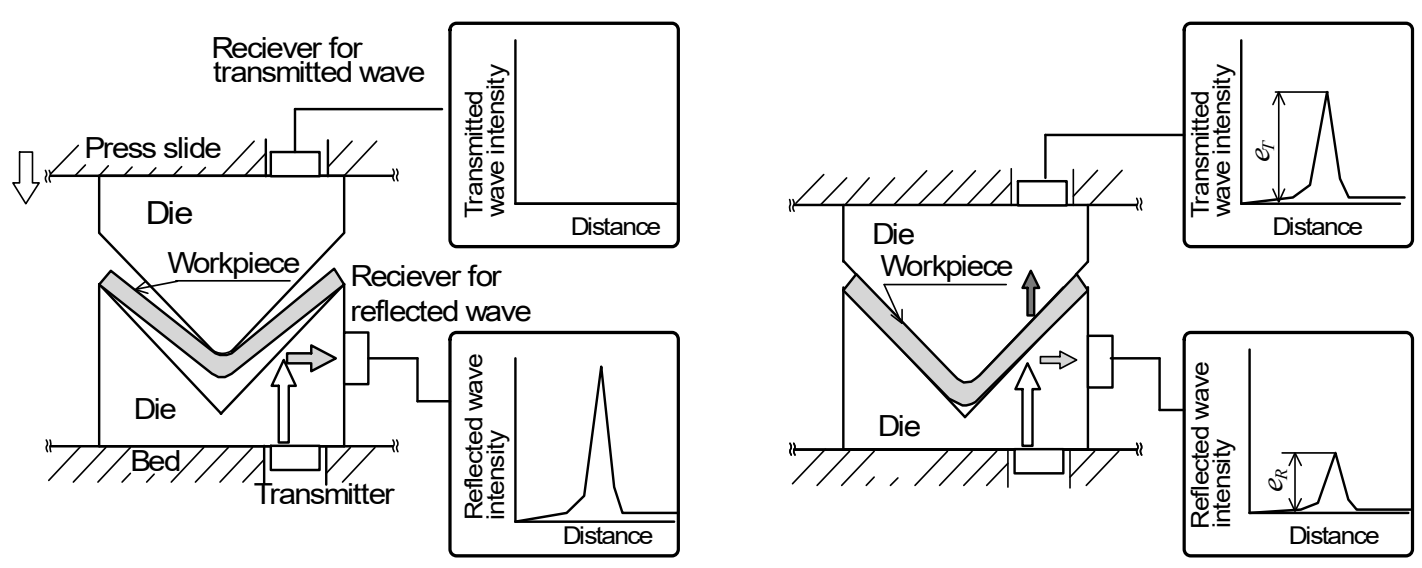

Fig. 2 Schematic of in-process monitoring for $\mathrm{V}$-shape stamping. 
Fig. 2 shows the procedure for in-process monitoring for V-shape stamping. When the press slide reaches the bottom dead center, the workpiece firmly attaches to the dies. As a result, the reflected wave intensity decreases and the transmitted wave intensity increases. The reflected wave height, $e_{R}$, and transmitted wave height, $e_{T}$, are defined as indicated in the figure. The authors have already established that the contact states could be measured by using these wave heights [4-6].

\section{Theoretical Formula for Elastic Waves in Metal Solids}

The ultrasonic waves sent out from the ultrasonic transmitter propagate in the metal solids as elastic waves without plastic deformation. When sound fields in the $y$-direction are uniform, the fundamental equations for elastic waves in the metal solid are expressed by Eqs. 3 and 4 [8-10]. Equation 3 describes Hooke's law and Eq. 4 describes particle motion. Here, $c_{11}, c_{33}, c_{55}$, and $c_{13}$ are components of a stiffness matrix. The materials used in this numerical analysis have isotropic elasticity, i.e., $c_{11}=c_{33}$ and $c_{55}=\left(c_{11}-c_{13}\right) / 2$. The $T_{x x}$ and $T_{z z}$ are normal stresses, and $T_{x z}$ is shear stress. The $\dot{u}$ and $\dot{w}$ are the velocity of the $x$ and $z$ directions.

$$
\begin{aligned}
& \frac{\partial}{\partial t}\left[\begin{array}{c}
T_{x x} \\
T_{z z} \\
T_{x z}
\end{array}\right]=\left[\begin{array}{ccc}
c_{11} & c_{13} & 0 \\
c_{13} & c_{33} & 0 \\
0 & 0 & c_{55}
\end{array}\right]\left[\begin{array}{c}
\frac{\partial \dot{u}}{\partial x} \\
\frac{\partial \dot{w}}{\partial z} \\
\frac{\partial \dot{u}}{\partial z}+\frac{\partial \dot{w}}{\partial x}
\end{array}\right] . \\
& \rho \frac{\partial}{\partial t}\left[\begin{array}{c}
\dot{u} \\
\dot{w}
\end{array}\right]=\left[\begin{array}{l}
\frac{\partial T_{x x}}{\partial x}+\frac{\partial T_{x z}}{\partial z} \\
\frac{\partial T_{z z}}{\partial z}+\frac{\partial T_{x z}}{\partial x}
\end{array}\right]
\end{aligned}
$$

We applied these to the velocity potential for Eqs. 3 and 4. The particle velocity vector is represented as Eq. $5[9,11]$ by using the velocity potential.

$$
\dot{\boldsymbol{u}}=\operatorname{grad} \Phi+\operatorname{rot} \boldsymbol{\Psi}
$$

This is where $\Phi$ is the scalar velocity potential, $\boldsymbol{\Psi}=\left[\Psi_{1}, \Psi_{2}, \Psi_{3}\right]$ is the vector velocity potential, and $\dot{\boldsymbol{u}}=[\dot{u}, \dot{v}, \dot{w}]$ is the particle velocity vector. We assumed that the sound fields in the $y$-direction were uniform in this study. Eqs. 6 and 7 are expressed from Eq. 5:

$$
\begin{aligned}
& \dot{u}=\frac{\partial \Phi}{\partial x}-\frac{\partial \Psi}{\partial z} . \\
& \dot{w}=\frac{\partial \Phi}{\partial z}+\frac{\partial \Psi}{\partial x} .
\end{aligned}
$$

where $\Psi$ is replaced by $\Psi_{2}$.

The potential equations Eqs. 8 and 9 are obtained from Eqs. 3, 4, 6, and 7:

$$
\begin{gathered}
\rho \frac{\partial \dot{\Phi}}{\partial t}=c_{11}\left(\frac{\partial \dot{u}}{\partial x}+\frac{\partial \dot{w}}{\partial z}\right) . \\
\rho \frac{\partial \dot{\Psi}}{\partial t}=c_{55}\left(\frac{\partial \dot{w}}{\partial x}-\frac{\partial \dot{u}}{\partial z}\right) .
\end{gathered}
$$


where $\dot{\Phi}$ is $\partial \Phi / \partial t$ and $\dot{\Psi}$ is $\partial \Psi / \partial t$. The $\rho \dot{\Phi}$ and $\rho \dot{\Psi}$ in Eqs. 8 and 9 have the units of stress [8]. The values of $\rho \dot{\Phi}$ and $\rho \dot{\Psi}$ describe the longitudinal and shear waves. The advantages of applying the velocity potential to the propagation of elastic waves are as follows $[9,11]$ :

1) Calculation load is minimized because the velocity potential is scalar.

2) Longitudinal and shear waves can be independently examined by using $\rho \dot{\Phi}$ and $\rho \dot{\Psi}$.

The elastic-wave equations to examine the acoustic fields in the die and the workpiece are solved by using the finite-difference time-domain (FDTD) method [7,8], which can be used to simultaneously analyze the reflected and transmitted waves. The elastic wave equations are transformed into difference equations in the spatial and time domains using the FDTD method.

For example, the difference equations of the velocity potential are expressed as:

$$
\begin{aligned}
& \rho \dot{\Phi}_{i, k}^{n+1 / 2}=\rho \dot{\Phi}_{i, k}^{n-1 / 2}+c_{11} \frac{\Delta t}{\Delta x}\left(\dot{u}_{i+1 / 2, k}^{n}-\dot{u}_{i-1 / 2, k}^{n}\right)+c_{11} \frac{\Delta t}{\Delta x}\left(\dot{w}_{i, k+1 / 2}^{n}-\dot{w}_{i, k-1 / 2}^{n}\right) . \\
& \rho \dot{\Psi}_{i-1 / 2, k-1 / 2}^{n+1 / 2}=\rho \dot{\Psi}_{i-1 / 2, k-1 / 2}^{n-1 / 2}+c_{55} \frac{\Delta t}{\Delta x}\left(\dot{w}_{i-1 / 2, k}^{n}-\dot{w}_{i-1 / 2, k-1}^{n}\right)-c_{55} \frac{\Delta t}{\Delta x}\left(\dot{u}_{i, k-1 / 2}^{n}-\dot{u}_{i-1, k-1 / 2}^{n}\right) .
\end{aligned}
$$

where $n$ is the time index and $i$ and $k$ are the $x$ and $z$ direction spatial indices.

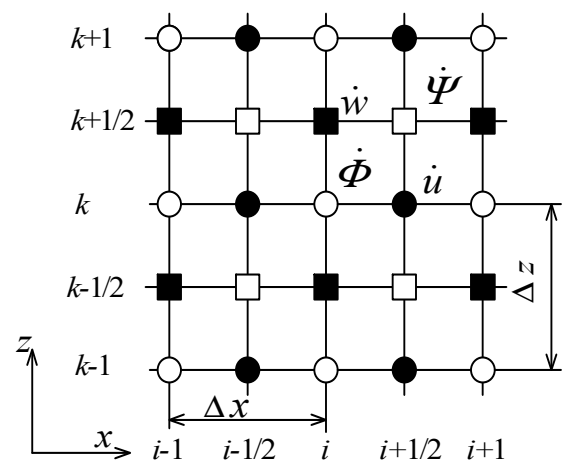

(a) Spatial lattice

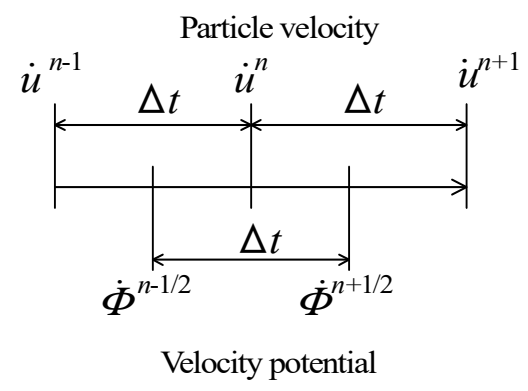

(b) Calculation procedure of velocity potential and particle velocity in time domain

Fig. 3 Spatial lattice and calculation procedure for FDTD simulations.

Fig. 3 outlines the spatial lattice and calculation procedure in the time domain. The velocity potential and particle velocities are set on difference lattice points. The velocity potential and particle velocity in the time domain are calculated alternately.

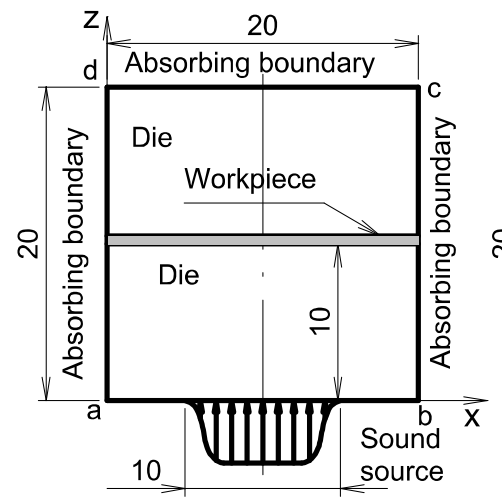

(a) Flat dies

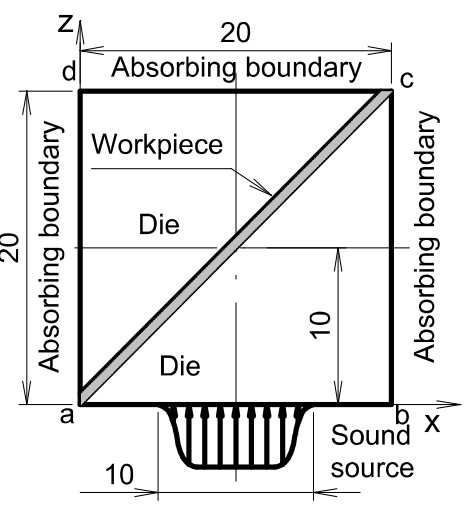

(b) V-shaped dies

Fig. 4 Calculation models for propagation of elastic waves around boundary surfaces of workpieces and dies.

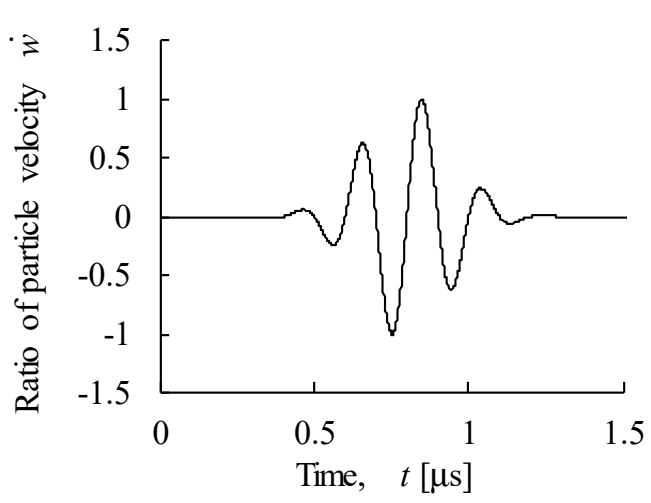

Fig. 5 Input Gaussian pulse.

The models used for calculating the acoustic fields on the basis of FDTD are outlined in Fig. 4. These calculation models were created to imitate the flat dies and V-shaped stamping dies. The distance between the sound source and the workpiece at the center of the model is $10 \mathrm{~mm}$. The 
workpiece and dies were assumed to have a continuous contact surface, and only their material properties differed. We used an aluminium thin plate as the workpiece. The material properties of the workpiece and the die used in the analysis are listed in Table 1, where $c_{l}$ and $c_{s}$ are the wave velocities of the longitudinal and shear waves. All materials are isotropic. The time step used for the FDTD $(\Delta t)$ is $2 \mathrm{~ns}$, and the spatial lattice interval is $\Delta x=\Delta z=20 \mu \mathrm{m}$. A sound source was placed at the bottom center of the calculation model and activated using the Gaussian pulse shown in Fig. 5. The frequency of the incident wave was $5 \mathrm{MHz}$. The boundary a-b of the calculation model was free, and the other boundaries (b-c, c-d, and c-a) were the absorbing boundaries. Mur's first-order absorbing conditions were applied as the absorbing boundary [10] to prevent unnecessary reflected waves from occurring.

Table 1 Materials used in numerical analysis of acoustic fields around workpiece and dies.

\begin{tabular}{|c|c|c|c|c|c|}
\hline \multirow{2}{*}{ Materials } & \multirow{2}{*}{$\begin{array}{c}\text { Longitudinal } \\
\text { elastic modulus } \\
E[\mathrm{GPa}]\end{array}$} & \multirow{2}{*}{$\begin{array}{c}\text { Density } \\
\rho\left[\mathrm{kg} / \mathrm{m}^{3}\right]\end{array}$} & \multirow{2}{*}{$\begin{array}{c}\text { Poisson's } \\
\text { ratio } \\
v[-]\end{array}$} & \multicolumn{2}{|c|}{ Acoustic velocity } \\
\hline & & & & $c_{l}[\mathrm{~m} / \mathrm{s}]$ & $c_{s}[\mathrm{~m} / \mathrm{s}]$ \\
\hline Steel ( S50C-JIS, dies) & 206 & 7850 & 0.29 & 5860 & 3190 \\
\hline $\begin{array}{c}\text { Aluminum } \\
\text { (A5052-JIS, workpiece) }\end{array}$ & 70 & 2960 & 0.33 & 5920 & 2980 \\
\hline
\end{tabular}

\section{Results and Discussion}

Fig. 6 and 7 indicate the propagation of ultrasonic waves at the boundary surface between a workpiece and a flat die. These results were calculated by using stress (Eqs. 3 and 4). The hydrostatic stress ratio, $\tau$, and the shear stress ratio, $\sigma$, are defined by:

$$
\begin{aligned}
T & =\left(T_{x x}+T_{z z}\right) / 2 . \\
\tau & =T / T_{0} . \\
\sigma & =T_{x z} / T_{0} .
\end{aligned}
$$

where $T$ is hydrostatic stress and $T_{0}$ is the maximum value of hydrostatic stress on the incident wave.
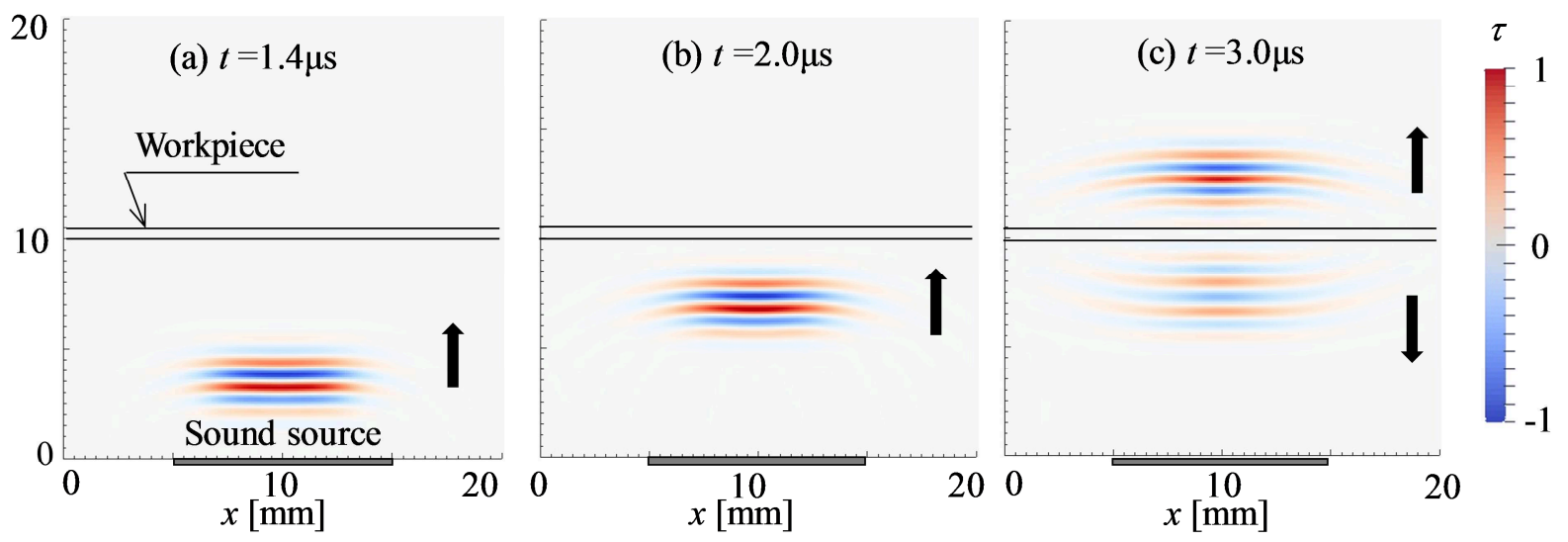

Fig. 6 Distribution of hydrostatic stress ratios.

The ultrasonic wave was incident from the sound source, which is the ultrasonic transducer (see Fig. 6). When the incident wave reached the workpiece, it was separated into the reflected wave and the transmitted wave at the boundary surface. The arrows in Fig. 6 are the directions of wave propagation. The reflected waves propagated to the opposite direction of the incident wave. The $\alpha$ waves indicated in Fig. 7(a) appeared at both corners of the sound source. The $\alpha$ and $\beta$ waves are propagated upward, although their velocities differ. The propagation velocity of the $\beta$ waves is the same as the acoustic velocity of the longitudinal waves, and that of the $\alpha$ waves is the acoustic velocity of the shear waves. Therefore, the wavelength of the $\alpha$ waves is shorter than that of the $\beta$ waves. Hence, it seems that the $\alpha$ waves are shear waves that are generated by the non-uniform 
distribution of input sound fields. On the other hand, the $\beta$ waves have the same wavelength as the longitudinal waves in Fig. 6 . We believe that the $\beta$ shear waves can be attributed to the propagation of the longitudinal waves. When ultrasonic waves passed through the workpiece, shear stresses $\gamma$ appeared in the upper die in Fig. 7 (c). The shear stresses in region $\gamma$ cannot be categorized into either longitudinal or shear waves in the shear stress ratios because the stress fields are very complicated. Thus, it was difficult to examine the separation of longitudinal and shear waves in the distribution of stress ratios.
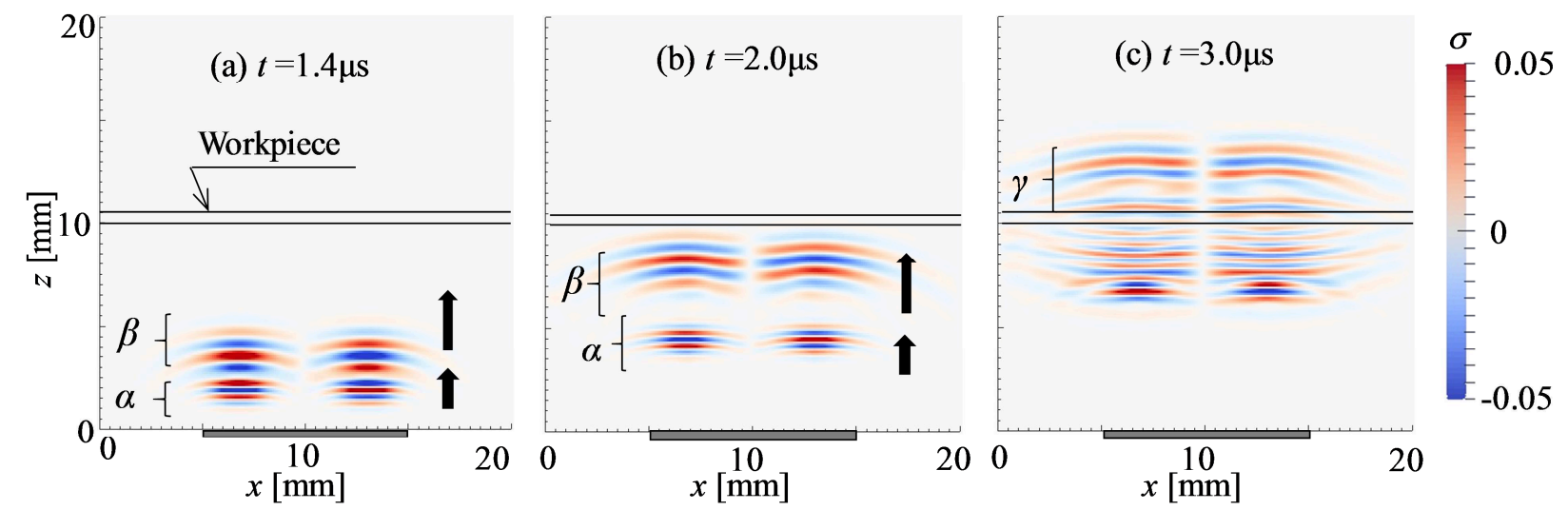

Fig. 7 Distributions of shear stress ratios.

Next, the acoustic fields were analyzed by using the vector potential. Fig. 8 and 9 show the numerical results that were calculated with the potential equations. The ratio of scalar velocity potential $P_{l}$ and the ratio of vector velocity potential $P_{S}$ in these figures are defined as:

$$
\begin{aligned}
& P_{l}=(\rho \dot{\Phi}) /\left(\rho \dot{\Phi}_{0}\right) . \\
& P_{S}=(\rho \dot{\Psi}) /\left(\rho \dot{\Phi}_{0}\right) .
\end{aligned}
$$

where $\rho \dot{\Phi_{0}}$ is the maximum value of $\rho \dot{\Phi}$ in the incident wave.

Since $P_{l}$ and $P_{s}$ independently describe each component of longitudinal and shear waves, they can be used to examine the propagation of the longitudinal and shear waves. The longitudinal incident waves in Fig. 8 at the boundary surface were either transmitted or reflected waves.
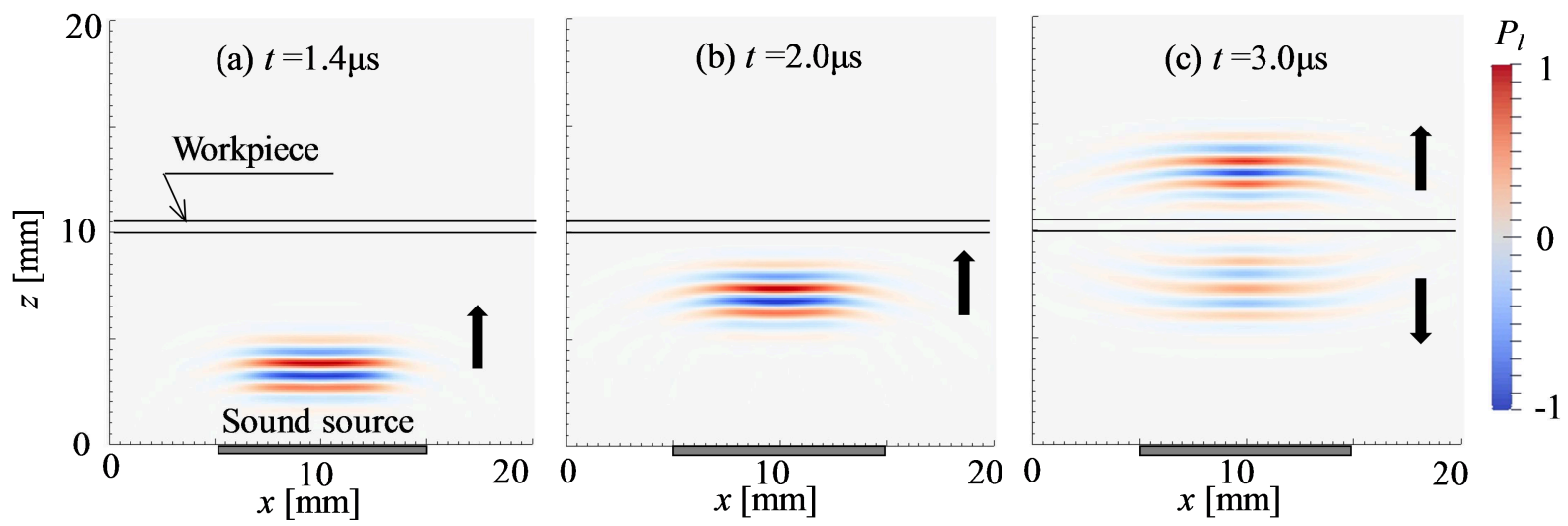

Fig. 8 Distribution of scalar velocity potential ratios.

We can only find the component of shear waves in Fig. 9. The $\alpha$ ' waves are shear waves. These waves are the same as $\alpha$ in Fig. 7(a). The $\beta$ waves in Fig. 7 did not appear in the distribution of vector velocity potential. The $\beta$ waves are not shear waves but rather waves excited by longitudinal waves. These results indicate that the incident elastic waves sent out from the sound source are mixtures of normal waves with circular or spherical wave fronts expanding from both sides of the transducer, and shear waves. 
At $t=3.0 \mu \mathrm{s}$, the shear waves (see $\gamma^{\prime}$ in Fig. 9(c)) appeared when the longitudinal waves passed through to the workpiece. These waves were shear waves that were converted from part of the incident longitudinal waves. Modal conversion occurred due to the oblique incidence of ultrasonic waves at the boundary surface of different media [10]. The wave front of the ultrasonic waves was uniform, and was circular on both sides of the waves. Hence, even if flat dies were used in stamping, the elastic waves sent from the ultrasonic transducer obliquely entered the workpiece. This oblique incidence was the reason that modal conversion occurred at the boundary surface of the flat dies. The results mean that elastic waves sent from an ultrasonic transducer were mixtures of normal waves with flat wave fronts, i.e., normal waves with circular or spherical wave fronts expanding from both sides of the transducer.
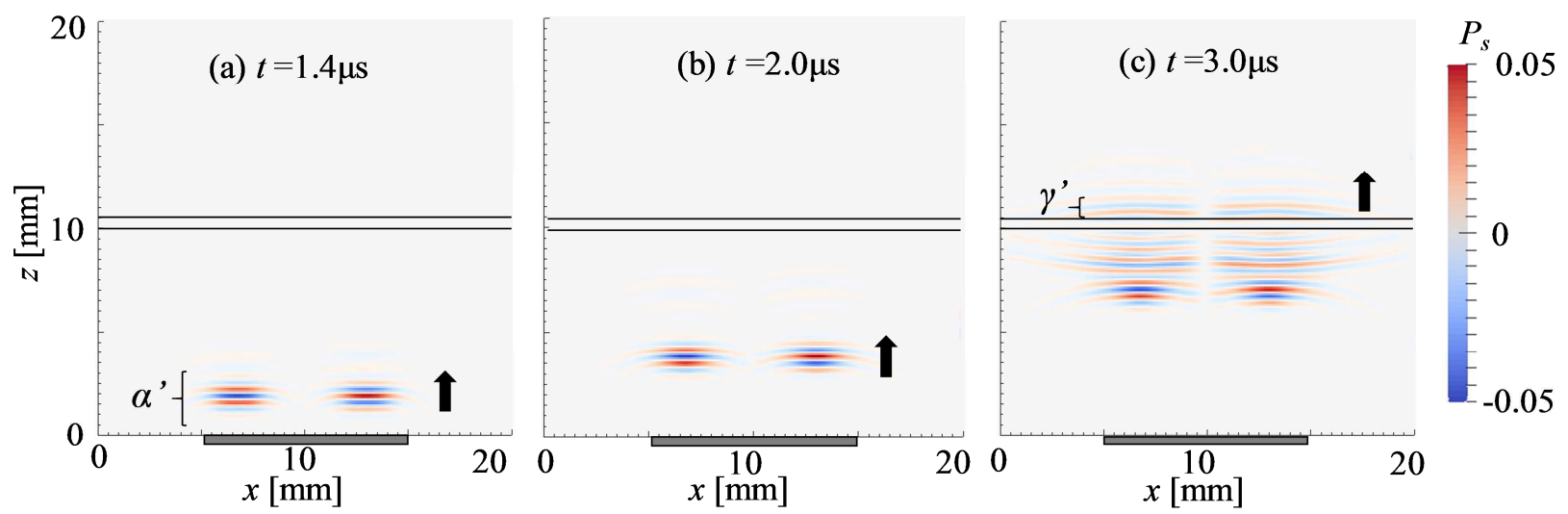

Fig. 9 Distribution of vector velocity potential ratios.

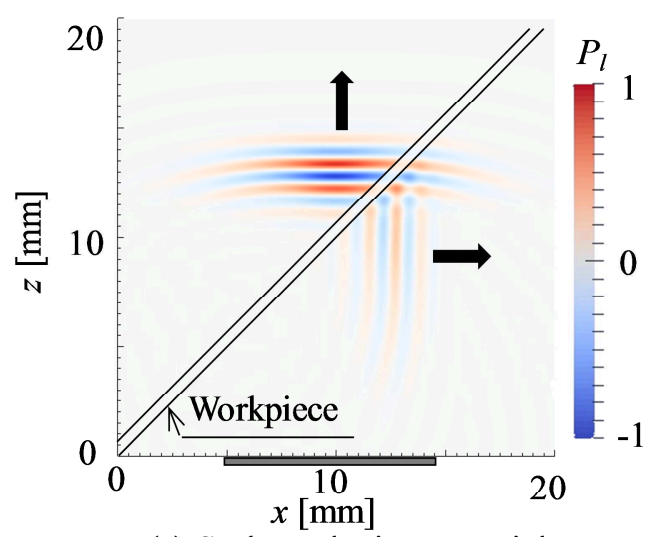

(a) Scalar velocity potential

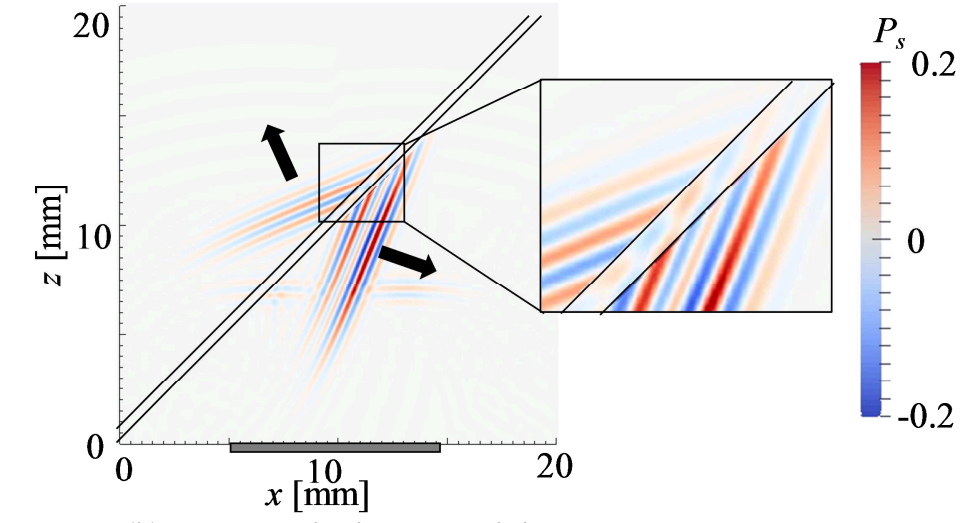

(b) Vector velocity potential

Fig. 10 Distribution of velocity potential ratios at $t=3.0 \mu$ s using $\mathrm{V}$-shaped stamping dies.

Next, we examined the propagation of ultrasonic waves near the inclined boundary surface between the workpiece and V-shaped stamping dies. Fig. 10 indicates the distribution of the velocity potentials near the inclined boundary surface. The elastic wave in Fig. 10(a) separated into a reflected or transmitted wave when the incident wave was incident at the workpiece. A longitudinal transmitted wave propagated in the direction of the incident wave. On the other hand, a longitudinal reflected wave was reflected at 45 degrees to the workpiece. When the ultrasonic wave passed through the workpiece, reflected and transmitted shear waves appeared near the inclined boundary surface between the workpiece and dies (see Fig. 10(b)). The maximum value of vector velocity potential was 0.15 . This value is more than 10 times that obtained using the flat dies. This is because the incident angle of ultrasonic waves for the boundary surface is large. The reflection angle and refraction angle were 23 degrees. Thus, no receiver placed in the direction of the incident waves could measure reflected and transmitted shear waves. It is necessary to adjust the transducer's position obtained from FDTD simulations to measure shear waves. The fields of vector velocity potential in workpieces are complicated, as indicated in Fig. 10. This is because ultrasonic waves are reflected and transmitted multiple times when they pass through thin plates during the stamping process. The synthetic waves 
of these waves were observed as reflected and transmitted waves. Thus, we believe that the distribution of reflected and transmitted waves became complicated in shape under the influence of multiple reflections and transmissions.

\section{Conclusion}

The authors used the finite-difference time-domain method to theoretically analyze the propagation of ultrasonic waves around the boundaries of workpieces and stamping dies. Key findings were:

(1) Modal conversion occurred at the die-workpiece boundary in such a way that normal waves with an inclined incident angle were transformed into normal and shear waves.

(2) Elastic waves sent from an ultrasonic transducer were mixtures of normal waves with flat wave fronts along the propagation path axis, normal waves with circular or spherical wave fronts expanding from both sides of the transducer, and shear waves.

(3) When a thin metal sheet was pressed between dies that had inclined stamping surfaces, ultrasonic elastic waves were reflected and transmitted multiple times.

\section{Acknowledgments}

This study was supported by the Amada Foundation, Japan, which the authors gratefully acknowledge.

\section{References}

[1] M. Masuko Y. Ito, Measurement of contact pressure by means of ultrasonic waves, Annals of CIRP XVII(3) (1968) 289-296.

[2] A. Takeuchi, Y. Kimura, T. Wakabayashi, T. Ishimaru, H. Mori, Early Detection of Abnormalities in Ball Bearings Using an Ultrasonic Technique, Trans. JSME Series C (in Japanese), 69-687 (2003), 3086-3091.

[3] A. Takeuchi, M. Satoh, M. Onozawa, Y. Sugawara, H. Aoki, Measurement of contact area using ultrasonic technique, Trans. JSME Series C (in Japanese), 65-640 (1999) 4767-4773.

[4] N. Hagino, J. Endou, S. Katoh, S. Okudera, M. Maruyama, M. Kubota, C. Murata, 2010. In-process monitoring for press forming, Steel Res. Int. 81(9) (2010) 674-677.

[5] N. Hagino, J. Endou, S. Katoh, S. Okudera, M. Maruyama, M. Kubota, Visualization of Contact Condition between Work-piece and Die during Stamping, Steel Res. Int. Special ed. 2011, (2011) 390-395.

[6] N. Hagino, J. Endou, S. Katoh, M. Ishihama, Visualization of Contact Condition between Work-piece and V-shape Die, Steel Res. Int. Special ed. 2012, (2012) 319-322.

[7] N. Hagino, J. Endou, S. Katoh, M. Ishihama, Propagation Behavior of Ultrasonic Wave around Boundary Surfaces of Workpieces and Dies, Procedia Eng. 81 (2014) 1073-1078.

[8] N. Hagino, S. Komiya, M. Ishihama, Propagation analysis of ultrasonic waves around boundary surfaces of between workpiece and V-shape stamping dies for in-process monitoring, Proc. IN-TECH2015 (2015), 1073-1078.

[9] M. Sato, Use of velocity potentials in the definition of absorbing boundaries for FDTD analysis of elastic wave fields, Acoustical Science and Technology, 24(6) (2003) 415-418.

[10] Y. Yamamoto, Ultrasonic wave basics engineering, Nikkan Kogyo Shinbun LTD., Tokyo, (1981).

[11] T. Kimura, K. Misu, S. Wadaka and M. Koike, Application of elastic FDTD method to simulation of acoustic fields in ultrasonic angle beam testing, IEICE Technical Report (in Japanese), 105-619 (2006) 11-16. 